\title{
Edge, fog and cloud computing in the cyber-physical systems networks
}

\author{
Andrey V. Gurjanov ${ }^{1}$, Anatoly G. Korobeynikov ${ }^{2}$, Igor O. Zharinov² and Oleg O. Zharinov ${ }^{3}$ \\ ${ }^{1}$ Director, Stock Company «Experimental Design Bureau «Electroavtomatika» named after P A Yefimov, 40, \\ Marshala Govorova St., Saint Petersburg, 198095, Russia \\ ${ }^{2}$ Faculty of Information Security and Computer Technologies, ITMO University, 49, Kronverksky Av., Saint \\ Petersburg, 197101, Russia \\ 3 Department of Problem-Oriented Computing Complexes, Saint Petersburg State University of Aerospace \\ Instrumentation, 67, Bolshaya Morskaia str., Saint Petersburg, 190000, Russia
}

\begin{abstract}
The data processing and technological tasks completion parallelism are the distributed control system base which use in a cyber-physical production edge, fog and cloud computing. The mutual agreement of operation and informative production processes is provided with a network message exchange technology supported with a functionally independent cyberphysical systems. To control a message queue and industrial equipment actions coordination they use communication system elements with data tide processing function in edge, fog and cloud network units and client applications placed in cyber-physical system devices. The industrial equipment own control processes are regulated with a local platform with edge computing. The expanded cyber-physical production technological task is controlled with a multi-process computing platform. To increase the quality of message in fact received with a cyber-physical system and to make faster its data processing is done with fog computing engaging equipment functional groups resources divided in a general information process. To transmit the technological task solution components into the network edge and to implement the fog computing of high parallelism degree in the production infrastructure is a perspective Industry 4.0 approach to organize the production systems interaction. They propose a cyberphysical production structure based on network technologies, which use edge, fog and cloud computing.
\end{abstract}

\section{Keywords}

Cloud computing, cyber-physical systems, Industry 4.0, information technologies

\section{Introduction}

The established industrial practices to construct a multi-service production infrastructure should be changed in a cardinal way today to reflect the Industry 4.0 basic tendencies. The cyber-physical systems (CPSs) connection and control aspects are a combination of information components integrated into the physical device, which is formed together with a monitor channel and production processes control channel $[1,2]$. The CPS informative components are computing and network units connected to a measuring system. This cyber-physical production (CPP) paradigm has some super-complicated system properties, which control is done through a network with restrictions justified with connection channels capacity $[3,4]$.

III International Workshop on Modeling, Information Processing and Computing (MIP: Computing-2021), May 28, 2021, Krasnoyarsk, Russia

EMAIL info@elavt.spb.ru (Andrey Gurjanov); korobeynikov_a_g@mail.ru (Anatoly Korobeynikov); mpbva@mail.ru (Igor Zharinov); zharinov73@inbox.ru (Oleg Zharinov)

ORCID: 0000-0003-0858-6619 (Andrey Gurjanov); 0000-0002-9968-0207 (Anatoly Korobeynikov); 0000-0003-2508-5939 (Igor Zharinov); 0000-0003-1219-8205 (Oleg Zharinov)

(c) (i) 2021 Copyright for this paper by its authors.

Use permitted under Creative Commons License Attribution 4.0
CEUR Workshop Proceedings (CEUR-WS.org) 
A task to control a CPS with communication restrictions today is the main researchers problem who develop theoretical solutions for closed dynamic systems, which characterize determined and stochastic production processes properties. Space and time restrictions influence all technological systems functionality where the control model quality indications include computing and message transportation expenses in a connection channel $[5,6]$. A delayed data transmission through a telecommunication network appears to be «swimming in time» CPS information justified, which violates the equal awareness principle of synchronically functioning technological equipment with operation closing state $[7,8]$.

The data «aging» makes it seriously indefinite for a CPP control, which has the automatics primary elements of homogeneous nature for industrial systems and telecommunication connection systems. Different CPS devices single network with a feedback engages the information exchange wireless channel in a control loop, which regulate measuring and computing operations of $[9,10]$ :

- the production processes technological structure agreed with the item manufacturing route programmed with the item digital twin;

- the CPSs technical structure to unite analytical machines into the smart factory production and testing divisions with virtual projections beyond physical devices;

- the CPS information network topology structure to provide equipment access to the company resources and data aggregation based on local connection channels and a system of gates.

The control technologies integration into telecommunication connection system let adapt discriminant-ly the CPS production processes using standardized agreement of the network maintenance level. The changing with time control signals reflect the production processes true state and are being transmitted through the connection channels with cyclic intervals [11]. The message transportation channel into CPP environment is an industrial automatics system element influencing the control processes. The feedback chain among CPSs as an information wireless network is an autonomous technological equipment control loops superstructure, which regulates remotely the production processes. The CPP control given target completion is based on computing and network technologies convergence, which may develop significantly the Industry 4.0 [12].

\section{The CPP computing concept}

The CPP informative development strategy requires to create the new IT (Information Technologies) architecture corresponding the production data operative processing principle right where the measurement took place. To change the existing technological systems for CPSs preserving the computing infrastructure is not an optimal option how to modernize the company production assets because of packages delivery time delays of huge variety, which appear in the connection channels. The more perfect technological solution is to distribute the CPP computing resources among periphery CPSs (fog and edge units) and cloud server equipment installed beyond the operational section [13, 14].

The distributed information system is a kind of compromise between computing platform productivity using advantages of CPS analytical capabilities and the centralized fog (cloud) environment. The resources division between the cloud and periphery CPSs with a centralized message transportation service under the industrial conditions reduces the connection channels load by reducing the being translated information volume and let organize computing directly in the network end points. The communication architecture of ultra-low traffic transmission delay provides the peripheral CPSs data concord, which are controlled with the connection channels. The quick CPS network response is important to support the crucially important CPP scenarios, analytical and reaction mechanisms, which are approximated to the real time functioning.

CPSs network unification with integrated intellectual units creates an IT architecture, which is not capable for operation and information processes of intense computing. An industrial CPS as a data source registered from different detectors generates a significant volume of information, which collection, local processing and systematizing must be done with analytical tools, which are from the network edge level. The preprocessed industrial content tide transmission combined with CPS intellectual equipment creates an alternative approach how to construct the CPP structure oriented for a cloud environment [15]. 
To initiate applications with a CPS provides services for a CPP adding functional capabilities of cloud services with a sufficient computing capability to control the peripheral equipment. The data local processing and storage create new CPP scaling standards how to send the traffic through an information network, which is relevant to control a CPS device. The technological solutions deployment based on the edge infrastructure computing role reinforcing increases the CPP reliability, which CPS network coordination is of low expenses for the data exchange channels with cloud resources.

An advanced solution to cover up CPP computing aspects requires to implement edge, fog and cloud technologies into information processes, which require network inter-machine CPS interaction. The computing level to which they transmit applications among CPP platforms depends on the data significance degree, which correspond the CPS control objective. The CPP services basic level provides self-controlling CPSs working through a wireless telecommunication network with the data exchange determined time characteristics.

Edge, fog and cloud computing execution engines form the core of the CPP management platform. The control server and peripheral nodes process the work (computational) load of the CPS, fragmented into technological subtasks. The network facilities of operating systems serving applications of edge CPSs and databases of fog and cloud data centers support informational multithreading of CPP associated with the order of receipt and processing of messages. Mass parallelism of cyber-industrial computing is achieved mainly by the joint use of CPS resources, eliminating duplication, and balancing the information load of technological systems operating in the network in the mode of remote nodes. The industrial standards underlying the computational concept of CPP are focused on the problems of network technologies used in the automatic control closed-loops of distributed information systems.

\section{The CPP computing structure}

The Industry 4.0 concept includes the distributed computing model implementation in the CPP components where the information connection between all sources and data consumers is done through general system technologies means engaging IT resources of the same type. The data processing and their network transmission to CPS is done with particular rules, which follow the computing structure scaling laws with horizontal and vertical hierarchy levels. The newest CPP computing technologies set important for distributed analytical systems creation includes:

- edge computing necessary for critical in time application completion functionality in terminal

CPS devices processing technological data vast amounts in the place of their first registering;

- fog computing necessary for volume tide traffic processing in the connection networks of low or predictable information transmission delays uniting end units and server equipment;

- cloud computing, where aggregated technological data is processed in data centers server equipment with applications working through virtual services.

The edge computing are completed with CPS physical devices resources with required productivity and reliability and CPP data processing algorithms without delays of local applications related to traffic transmission to the network. The CPS autonomous platform capabilities as for edge computing are used to support applications monitoring the plant technological processes, which are done in integrated work chambers. To increase the peripheral analytics quality is justified with data processing in time approximated to the real one.

The fog computing are additional CPP automatizing components to shorten the CPS waiting time to process the network requests. Somewhere in between and server equipment there is a CPS interaction level to provide the application dynamic adaptation to the network conditions with the standard operation service without any duplication of CPS computing functions. To make the data processing processes to be parallel in the CPS edge units and in the fog data center establishment may reduce the passing through capability requirements for the workshop connection channel and may increase making a decision speed to detect a deviation from the established norms of the being controlled indications. The fog data center establishment is a computing structure section, which puts in agreement operation and information edge CPSs work processes with control means and data processing means while they have their own services.

The cloud computing are done with centralized data centers resources to provide the CPS telemetry processing. The resources and information packages distribution within the cloud infrastructure is done 
with the communication equipment, which grants the CPS the universal network access. The local networks edges traffic transmission to the cloud servers are routed with network protocol system and gates with integrated controllers. Data and connection processing as much as application control is done with general production computing infrastructure to monitor the edge CPSs and fog data centers network activity. The CPP computing structure is given in Figure 1:.

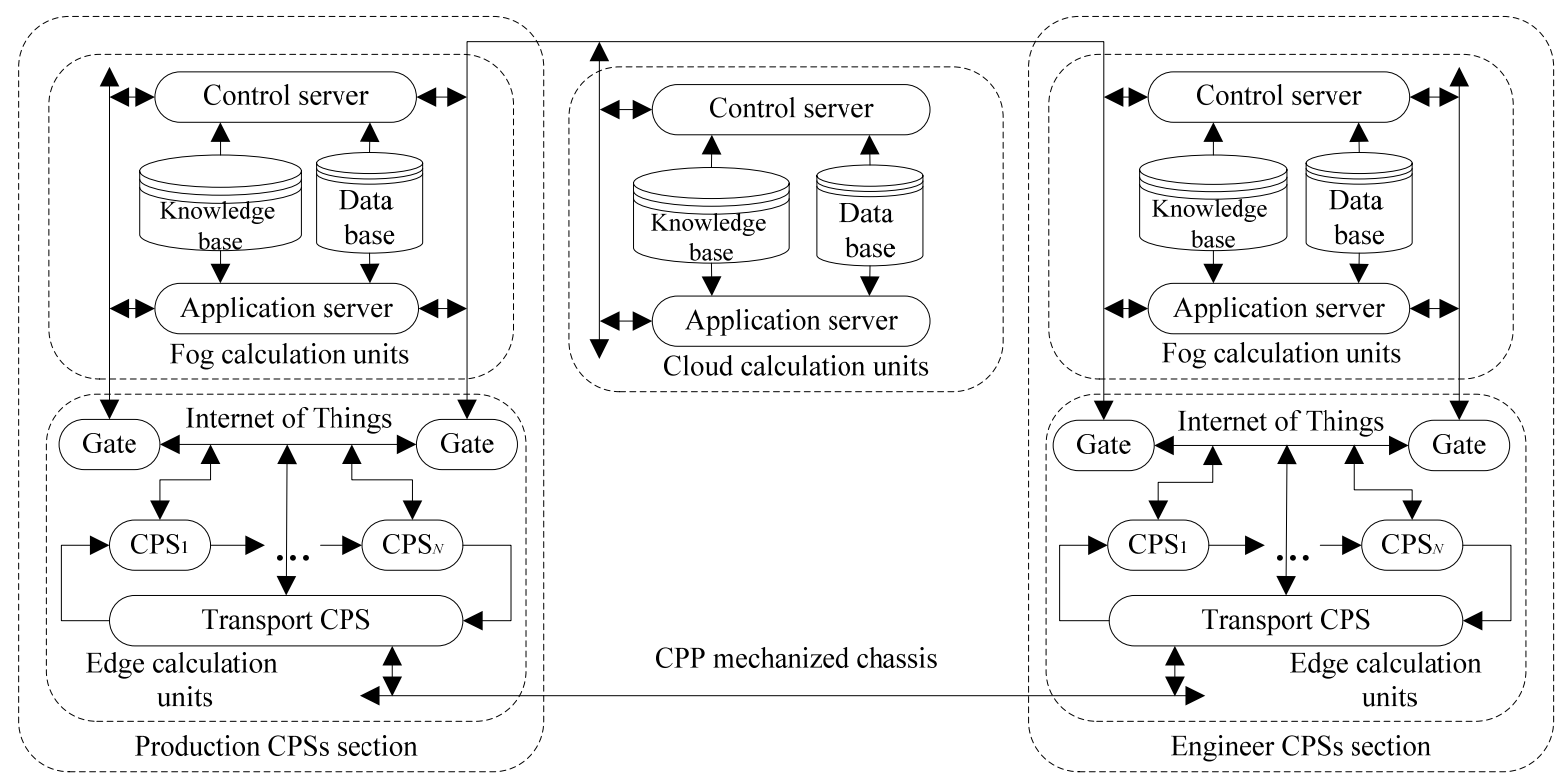

Figure 1: The CPP computing structure

The computing technologies combination in the CPP infrastructure leads to creation of edge CPS devices network connected with a fog architecture through a system of gates with cloud servers. The fog architecture is a computing bridge between data generation and data pre-processing points and remote points where the applications are placed to control a CPP. The load distribution among edge elements, fog ones and cloud computing are done with operation system means, which let configure the company information network with a program.

The network computing technologies of CPP machine infrastructure combines physically unconnected CPSs into an IT services environment concentrated among data centers fog and cloud data analysis to reach a general goal how to control the industrial object infrastructure. The edge CPSs information processes and interaction with each other are synchronized with net services optimizing link protocols among applications placed in the technological systems calculation platform. The data tides distribution is done with a number of operational systems connected to a CPS through the network having a low relativity of technological data computing processes and for control commands.

The heterogeneous CPS communication with different net domains united in groups supporting independent and distributed computing processes using API (Application Program Interface)-oriented connection technologies. The CPP exchange digital channels system engages wired (optical fiber) connections of control systems based on Ethernet and separate wireless nets for IoT (Internet of Things)-applications. The CPP net system control element implements human participation with a digital avatar into the industrial object infrastructure to solve non-interactive technological tasks.

Net technologies supporting edge, fog and cloud calculation platforms are of the distributed information system elements where the technological data processing is done in parallel in physical and virtual environments. The CPS connection with several calculation units among themselves and with data centers is done with network channels, which information packages transmission time corresponds the Data rate theorem requirements. The network computing exchange density is defined with technological processes control system properties and CPS machine interfaces characteristics, which in parallel and independently complete the CPP processes. 


\section{Conclusion}

New requirements of the CPP technologies justify the task solution necessity how to unite CPS computing capabilities and server equipment based on connection technology. To organize CPS interaction into network environment is the first priority task to project technological systems optimal control laws presented in the CPP infrastructure with physical and virtual resources. The calculator integration weak point is the data transmission channel, which has restrictions of passing through ability within a single address network company space.

The effective ways to control traffic and computing with appeared in network loads related to the analytical functions transition to the peripheral CPS platforms, which are information system edge units inside the CPP. The technological data processing and storage in intermediary edge (fog) network units reduce significantly cloud servers computing load and provides CPP resources dynamic control with an automatic anti-failure property.

To reconfigure and cluster the CPSs in the CPP processes without them being interrupted they apply new computing concept, which combine approaches to distribute and localize technological data processing resources in three levels presented as edge network segment, fog architecture and centralized cloud infrastructure. The CPP computing capabilities three levels classification is based on the concepts, which add each other and provides technological data transmission and analysis without any network delays. The production information is aggregated in different network points placed in between its edge and the cloud and which is translated through a lot of connection channels functioning in parallel.

The autonomous CPP calculators coordination is provided with data prioritizing mechanisms, which are relevant for the current technological task being completed. The computing infrastructure active scaling engages network and operation services, which systematize data packages and segment the traffic according to the cyber-physical production CPS terminal devices being used. The fog computing support platform is an additional information processing mean to expand cloud technologies edges until the network units placed very close to the technological data generation place. Calculators installed in the network periphery may autonomously process the data under unstable link channel connection conditions to increase CPP general reliability.

The local and remote computing resources industry application makes the data transmission protocols and interfaces unifying problem to be actual with an ability of CPS network quick response different in the equipment technical specifications and software components. But making network scheme more complicated and dynamic changes of its structure depend on the CPP computing load requires the implementation an increased number of routers, gates and routers to the company information system but also they have to develop the new type of operation system and applications, which functioning specifics is required with the Industry 4.0 necessities. The CPP computing resources scaling is done through the network elastic property with a sufficient passing through ability to create a technological processes and equipment control closed-loop system represented as CPS class.

\section{References}

[1] M. Abbasi, M. Yaghoobikia, M. Rafiee, A. Jolfaei, M. R. Khosravi, Computer communications 153 (2020) 217-228.

[2] R. Mahmud, S. N. Srirama, K. Ramamohanarao, R. Buyya, Journal of parallel and distributed computing 135 (2020) 177-190.

[3] G. Caiza, M. Saeteros, W. Onate, M. V. Garcia, Heliyon 6(4) e03706 (2020).

[4] P. Verma, S. K. Sood, H. A. Kaur, Microprocessors and microsystems 72102929 (2020).

[5] M. Dabbaghjamanesh, A. Moeini, A. Kavousi-Fard, A. Jolfaei, Journal of parallel and distributed computing 146 (2020) 15-24.

[6] L. Bulej, T. Bures, A. Filandr, P. Hnetynka, I. Hnetynkova, J. Pacovsky, G. Sandor, I. Gerostathopoulos, Journal of systems and software 172110872 (2021).

[7] N. Verba, K-M. Chao, J. Lewandowski, N. Shah, A. James, F. Tian, Future generation computer systems 91 (2019) 48-60.

[8] S-Y. Lin, Y. Du, P-C. Ko, T-J. Wu, P-T. Ho, V. Sivakumar, R. Subbareddy, Computer communications 160 (2020) 636-642. 
[9] J. Zietsch, M. Vogt, B D. Lee, S. Thiede, CIRP Journal of manufacturing science and technology 31 (2020) 351-369.

[10] D. A. Zakoldaev, A. G. Korobeynikov, A. V. Shukalov, I. O. Zharinov, IOP Conference Series: Materials Science and Engineering 665(1) 012015 (2019).

[11] K-C. Chang, K-C. Chu, H-C. Wang, Y-C. Lin, J-S. Pan, Future generation computer systems 108 (2020) 445-453.

[12] A G. Korobeynikov, M. E. Fedosovsky, I. O. Zharinov, V. I. Polyakov, A. V. Shukalov, A. V. Gurjanov, S. A. Arustamov, Advances in intelligent systems and computing 680 (2018) 5056.

[13] B. D. Deebak, F. AL-Turjman, Future generation computer systems 116 (2021) 406-425.

[14] N. Kumar, A. V. Vasilakos, K-K. Choo, L. T. Yang, Future generation computer systems 105 (2020) 754-756.

[15] T. Coito, M. S. Martins, J. L. Viegas, B. Firme, J. Figueiredo, S. M. Vieira, J. M. Sousa, Computers in industry $123103329(2020)$. 